\title{
A systematic review and meta-analysis of clinical research on treating angina pectoris of coronary heart disease with traditional Chinese medicine to promote blood circulation and remove blood stasis
}

\author{
Wen Chen ${ }^{1 \#}$, Bijuan Wang ${ }^{2 \#}$, Yanzhi Ge ${ }^{3}$, Haipeng Xü ${ }^{2}$, Chunyan Jiang ${ }^{4}$, Pengfei Yu ${ }^{5}$, Xian Zhang ${ }^{1}$, \\ Junfang Yang ${ }^{1}$, Haoran $\mathrm{Hu}^{1}$, Hong Song ${ }^{1}$ \\ ${ }^{1}$ School of Basic Medicine, Zhejiang Chinese Medicine University, Hangzhou, China; ${ }^{2}$ The Third Clinical Medical College, Zhejiang Chinese \\ Medical University, Hangzhou, China; ${ }^{3}$ The First Clinical Medical College, Zhejiang Chinese Medical University, Hangzhou, China; ${ }^{4}$ College of \\ Traditional Chinese Medicine, Zhejiang Pharmaceutical College, Ningbo, China; ${ }^{5}$ Department of Traditional Chinese Medicine, Yuhang District \\ Third People's Hospital Liangzhu Branch, Hangzhou, China \\ Contributions: (I) Conception and design: All authors; (II) Administrative support: All authors; (III) Provision of study materials or patients: All \\ authors; (IV) Collection and assembly of data: All authors; (V) Data analysis and interpretation: All authors; (VI) Manuscript writing: All authors; (VII) \\ Final approval of manuscript: All authors. \\ "These authors contributed equally to this work. \\ Correspondence to: Hong Song. School of Basic Medicine, Zhejiang Chinese Medicine University, Zhejiang University of Traditional Chinese

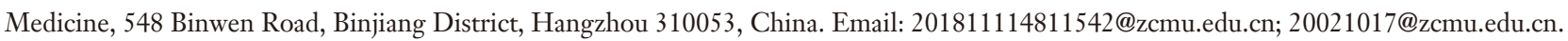

Background: Research has shown that traditional Chinese medicine (TCM) can achieve good results in the treatment of angina pectoris. In this study, we aimed to explore the therapeutic effect of TCM in the treatment of angina pectoris of coronary heart disease (CHD) through a literature search and meta-analysis.

Methods: The PubMed, Embase, CBM (China Biology Medicine) Web of Science databases were searched for studies on the treatment of angina pectoris of CHD with TCM. Inclusion and exclusion criteria were applied, and high-quality articles published from 2010.1 to 2021.8 were selected. The RevMan 5.3.5 software was used to evaluate the therapeutic effect indicators of TCM.

Results: Nine studies involving 824 patients were included in the meta-analysis, and the overall risk of literature bias was low. The results of meta-analysis showed that compared with conventional Western medicine, TCM + conventional Western medicine had a better efficacy indicators of angina pectoris using the fixed-effects model [odd rate $(\mathrm{OR})=3.20,95 \%$ confidence interval $(\mathrm{CI})$ : $(2.09,4.90), \mathrm{Z}=5.35, \mathrm{P}<0.00001$ ]. The frequency of angina pectoris was measured by random-effects model, and the statistical results were [standard mean difference $(\mathrm{SMD})=-1.85,95 \% \mathrm{CI}:(-2.29,-1.41), \mathrm{Z}=8.22, \mathrm{P}<0.00001]$. The adverse events was measured by fixed-effects model, and the statistical results were [OR $=0.48,95 \% \mathrm{CI}:(0.21,1.08), \mathrm{Z}=1.78$, $\mathrm{P}=0.08]$.

Discussion: The application of TCM in the treatment of angina pectoris of CHD can improve the therapeutic effect, reduce the frequency of angina pectoris, shorten the attack time, reduce serum total cholesterol, and improve the quality of life after treatment, but it has no obvious reducing effect on blood lipids.

Keywords: Traditional Chinese medicine (TCM); coronary heart disease (CHD); angina pectoris; meta-analysis

Submitted Jul 27, 2021. Accepted for publication Sep 18, 2021.

doi: $10.21037 /$ apm-21-2233

View this article at: https://dx.doi.org/10.21037/apm-21-2233 


\section{Introduction}

Coronary heart disease (CHD), also known as coronary atherosclerotic heart disease, is a heart disease which occurs when blood vessels narrow due to atherosclerosis and myocardial ischemia (1). Due to the insufficient blood supply of coronary artery, there is a sharp transient ischemia and hypoxia in myocardium, which result in the symptoms of palpitations, fatigue, chest pain, accompanied by systemic fever, panic, vomiting (2). At present, the treatment of angina pectoris is mainly focusing on dilating blood vessels, improving myocardial ischemia, inhibiting platelet aggregation, and resolving arteriosclerotic plaques. However, long-term drug treatment can entail side effects such as liver and kidney injury (3).

Traditional Chinese medicine (TCM) therapy has its unique advantages in the treatment of CHD related symptoms of angina pectoris, from the traditional Chinese medicine knowledge, angina pectoris belongs to the cognitive category of "chest obstruction" and "heartache", and its final pathogenesis is heart blood stasis. Therefore, promoting blood circulation to remove blood stasis should be its main treatment (4). A study (5) applied the combination of TCM and conventional Western medicine in the treatment of angina pectoris, achieving encouraging results. Another study (6) divided 102 angina pectoris patients into two groups, one with the treatment of a special Chinese herb decoction, the other with the treatment of nifedipine, isosorbide dinitrate, and enteric solubility aspirin, the result showed no significant difference in terms of angina pectoris symptoms improvement, the cardiac function index and the blood lipid index for the Chinese decoction group even better. A large number of such kind of randomized controlled studies (RCTs) have been conducted, but these studies have variable quality and a lack of systematic review. In this study, a systematic review and meta-analysis of such studies were conducted to investigate the therapeutic effect of TCM in the treatment of angina pectoris of CHD, all of which had the intervention through oral TCM administration and most of them based on the treatment of conventional medicine like phospholipid, Breceptor blocker, or calcium channel blocker. We present the following article in accordance with the PRISMA reporting checklist (available at https://dx.doi.org/10.21037/apm-21-2233).

\section{Methods}

\section{Databases}

PubMed, Embase, CBM (China Biology Medicine
Database) and Web of Science were selected as the database sources for literature in this study.

\section{Search strategy}

The keywords "traditional Chinese medicine", "decoction", "TCM", "angina pectoris", and "coronary heart disease" were used to search literature published in the abovementioned databases from 2010.1 to 2021.8 .

\section{Inclusion and exclusion criteria of literature}

\section{Inclusion criteria}

The inclusion criteria for the meta-analysis were as follows: (I) single-center or multi-center RCTs; (II) the study participants had CHD and a definite diagnosis of angina pectoris; (III) the study participants were divided into a control group and an intervention group for comparative analysis; the intervention group was treated with a combination of TCM for promoting blood circulation and removing blood stasis (including decoction, pills, capsules, etc.) and Western medicine, and the control group was treated with conventional treatment (including simvastatin, isosorbide mononitrate, aspirin, or other commonly used Western medicines).

\section{Exclusion criteria}

The exclusion criteria for studies were as follows: (I) observational studies, investigations, guidelines, reviews, case analyses, and other studies; (II) studies with a sample size of less than 40; (III) studies without observation indicators, or studies with outcomes of incomplete or conflicting data.

\section{Literature screening}

Two retrieval personnels checked the database search results independently, remove the repetitive literatures. Preliminary screening was performed according to the inclusion and exclusion criteria by reading the abstracts of the RCTs. Then the full texts of the potentially eligible RCTs were read. Finally, the eligible studies were included for meta-analysis after quality evaluation.

\section{Literature quality evaluation}

The Jadad scoring criteria (7) were used to evaluate the quality of the included RCTs, and studies with fewer than 3 points were excluded. The evaluation process was independently carried out by two researchers. In cases 
Table 1 Outcome measures of the included studies

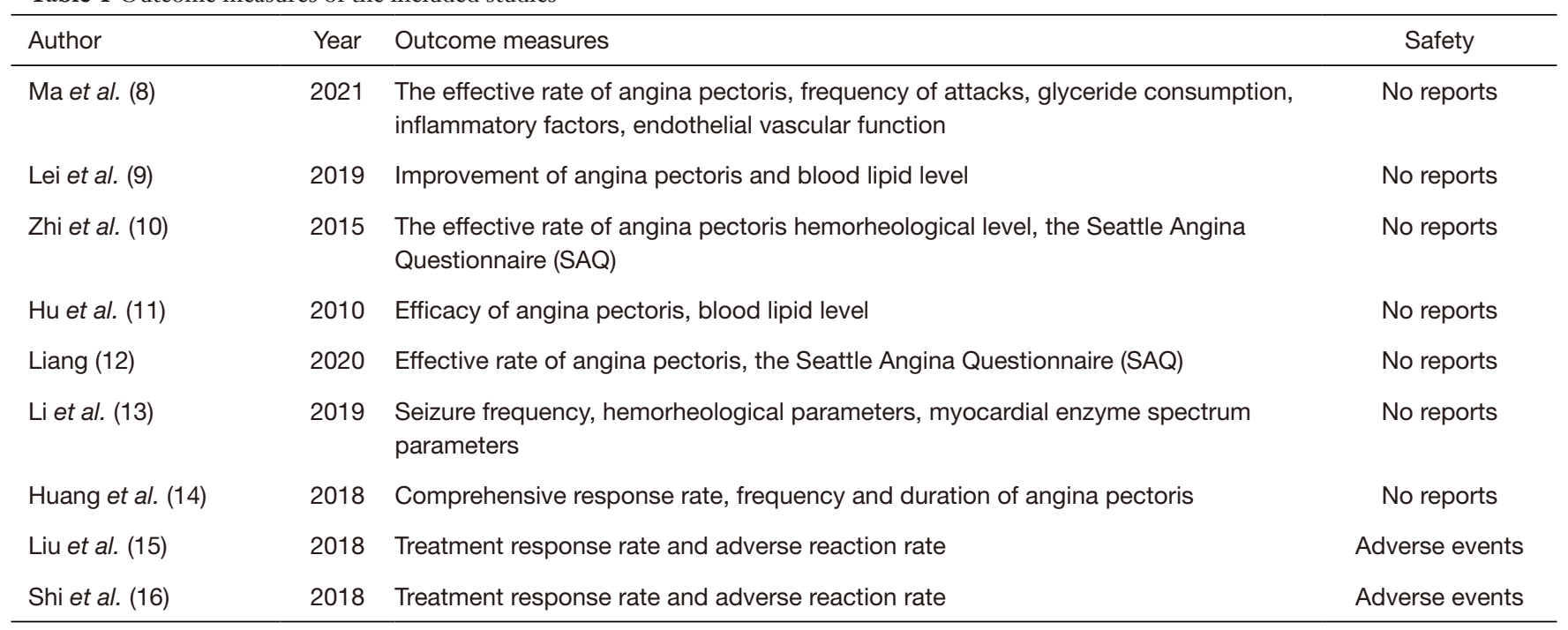

of conflicting opinion, a third researcher was invited to participate as an arbitrator.

\section{Data extraction}

The full texts were read by a researcher, who extracted data, including interventions, total number, grouping, characteristics of study participants, and outcome measures, and entered them into an Excel sheet.

\section{Outcome measures}

All outcome measures were collected from the included articles and are shown in Table 1.

\section{Statistical methods}

The RevMan 5.3.5 software (issued by the Cochrane Collaboration) was used to perform a comprehensive analysis to investigate the efficacy, the frequency of angina pectoris attack, blood lipid level, quality of life score, and other indicators after angina pectoris treatment. All percentage data were converted to the actual number of objects. Binary statistics were reported as OR and $95 \% \mathrm{CI}$, and continuous statistics were reported as SMD and 95\% CI. Differences were considered statistically significant with $\mathrm{P}<0.05$.

\section{Literature bias risk and heterogeneity}

The RevMan 5.3.5 software was used to assess the bias risk of the included RCTs. The studies were assessed in the following six aspects: the generation of sequences, classification concealment, blinding method, outcome measures, selective reporting, and other biases. The bias risk was included in the calculation of the final statistics and the preparation of forest plots. $\mathrm{I}^{2}$ analysis and $\mathrm{Q}$ check were performed to assess the heterogeneity of the included studies. Heterogeneity was indicated by $\mathrm{I}^{2}>50 \%$ or $\mathrm{P}<0.1$, in which case the random-effects model was used. In the absence of heterogeneity, the fixed-effects model was used. When heterogeneity existed between studies, eliminating literature one by one to find which caused the heterogeneity. The fixed-effects model and random-effects model switch were used to conduct the sensitivity analysis, if the result showed both no significant difference, the results were stable. Funnel plots were used to represent publication bias.

\section{Results}

\section{Literature screening results}

In this study, 117 studies were initially screened. According to the inclusion and exclusion criteria, and the literature quality evaluation, 9 studies involving 824 patients were finally included in the meta-analysis, as shown in Figure 1. The basic characteristics of the included studies are shown in Table 2.

\section{Literature bias and quality assessments}

As shown in Figure 2 and Figure 3, the quality of the 


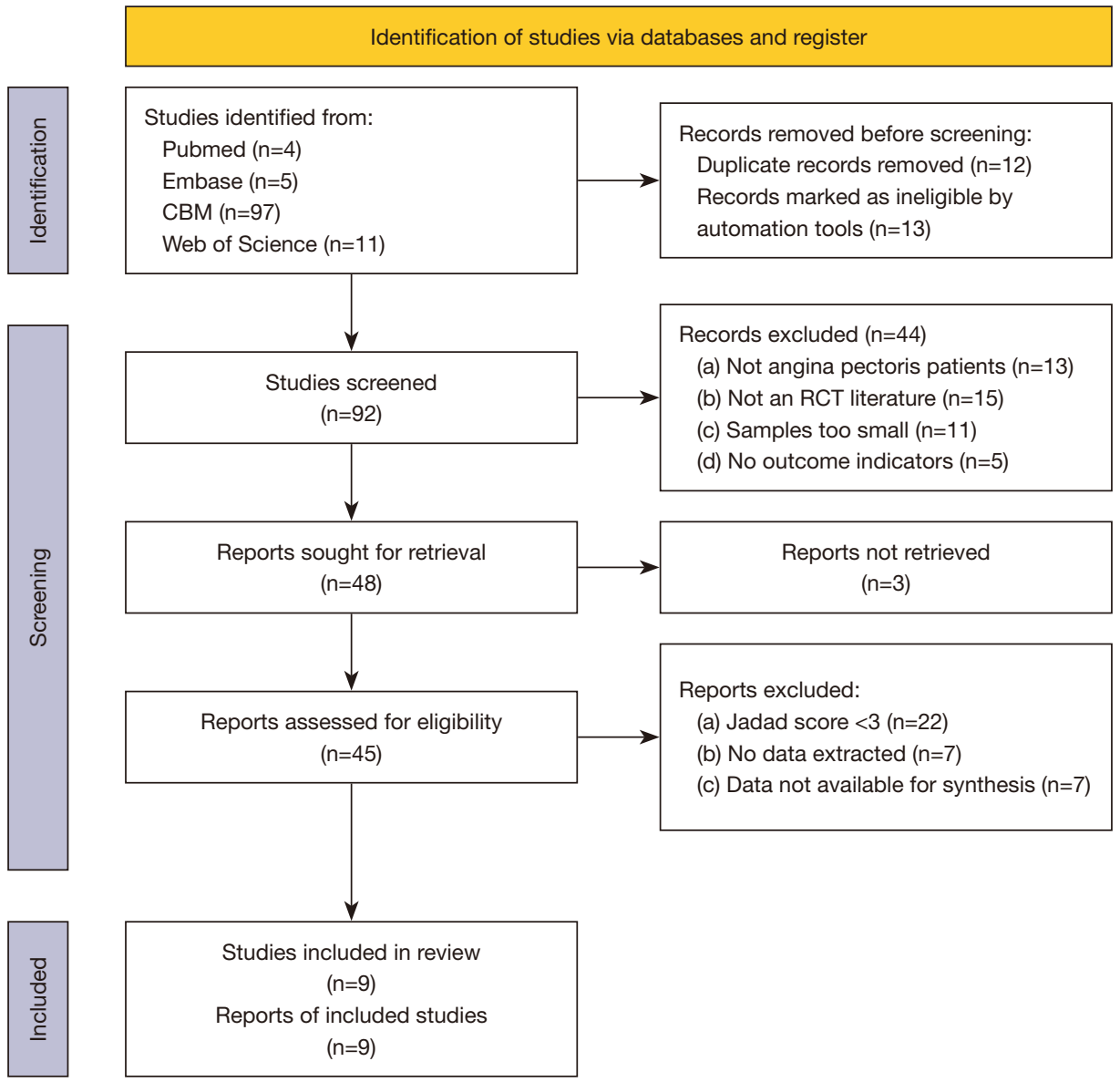

Figure 1 The selection flowchart. CBM, China Biology Medicine; RCT, randomized controlled studies.

Table 2 Basic characteristics of the included studies

\begin{tabular}{|c|c|c|c|c|c|c|c|c|}
\hline Author & Year & $\begin{array}{c}\text { Number } \\
\text { (T/C) }\end{array}$ & $\begin{array}{l}\text { Male } \\
(\mathrm{T} / \mathrm{C})\end{array}$ & $\begin{array}{c}\text { Mean age } \\
\text { (years) (T/C) }\end{array}$ & $\begin{array}{l}\text { Intervention group } \\
\text { treatment }\end{array}$ & $\begin{array}{l}\text { Control group } \\
\text { treatment }\end{array}$ & $\begin{array}{l}\text { Observation } \\
\text { period (days) }\end{array}$ & $\begin{array}{l}\text { Jadad } \\
\text { score }\end{array}$ \\
\hline Ma et al. (8) & 2021 & $47 / 46$ & $29 / 27$ & $61.3 / 60.7$ & TCM + Western medicine & Western medicine & 56 & 4 \\
\hline Lei et al. (9) & 2019 & $20 / 20$ & $10 / 9$ & $63.6 / 61.3$ & TCM + Western medicine & Western medicine & 42 & 5 \\
\hline Zhi et al. (10) & 2015 & $52 / 52$ & $30 / 29$ & $56.6 / 56.5$ & TCM + Western medicine & Western medicine & 40 & 6 \\
\hline Li et al. (13) & 2019 & $63 / 63$ & $31 / 29$ & $68.3 / 70.5$ & TCM + Western medicine & Western medicine & 56 & 4 \\
\hline Huang et al. (14) & 2018 & $36 / 35$ & $22 / 21$ & $52.6 / 51.5$ & $\mathrm{TCM}+$ Western medicine & Western medicine & 60 & 5 \\
\hline Liu et al. (15) & 2018 & $69 / 69$ & $36 / 34$ & $61.3 / 62.9$ & TCM + Western medicine & Western medicine & 14 & 6 \\
\hline
\end{tabular}

T represents the intervention group; C represents the control group. TCM, traditional Chinese medicine. 


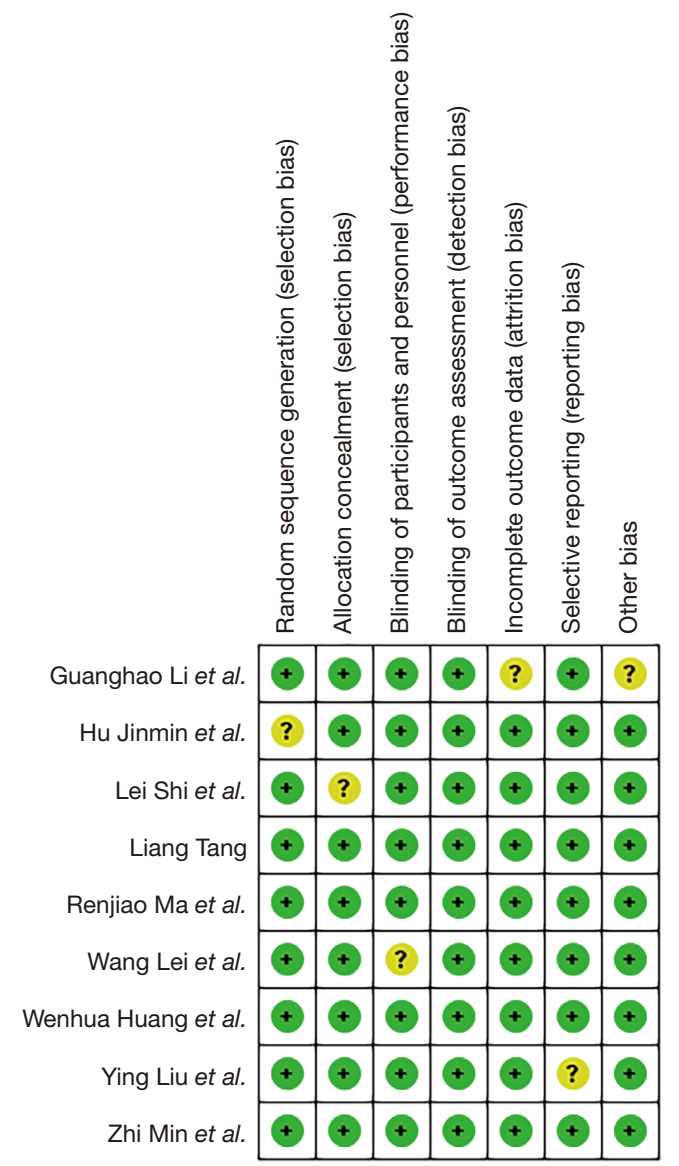

Figure 2 Quality assessment of the 9 included articles performed with RevMan.

9 articles included in this study was evaluated according to the Cochrane Random Intervention Evaluation System. The random sequence generation method was not explained in 1 study; the classification concealment was unclear in 1 study; the blinding method was unclear in 1 study; the outcome index data was incomplete in 1 study; and for 1 study, there was unknown bias. Nevertheless, the overall risk of bias of the 9 articles was low.

\section{Meta-analysis results}

\section{Effective rate}

Of the 9 articles finally included, Li et al.'s study (13) did not report effective rate indicator, so only 8 articles were elected into the synthesis. The results showed that the 8 articles exhibited statistical homogeneity $\left(\mathrm{I}^{2}=25 \%, \mathrm{P}=0.23\right)$, and the effective rate of the intervention group was greater than that of the control group [OR $=3.20,95 \% \mathrm{CI}$ : $(2.09$,
4.90)], the statistical value was $\mathrm{Z}=5.35, \mathrm{P}<0.00001$, and the difference was statistically significant. These results indicated that the TCM + conventional Western medicine group exhibited a significantly greater therapeutic effect than the conventional Western medicine treatment group (Figure 4).

\section{Angina attack frequency (times)}

Three studies reported the frequency of angina pectoris attack as an outcome indicator. Meta-analysis showed revealed statistical heterogeneity between the 3 studies $\left(\mathrm{I}^{2}=59 \%, \mathrm{P}=0.09\right)$, so the random-effects model was used. Angina attack was less frequent in the intervention group than the control group [SMD $=-1.85,95 \%$ CI: $(-2.29$, $-1.41)$, the statistical value was $Z=8.22, P<0.0001$, and the difference was statistically significant. These results indicated that TCM + conventional Western medicine had a significantly lower frequency of angina pectoris attack in patients comparing to the conventional Western medicine (Figure 5).

\section{Analysis of other indicators}

As shown in Table 3, meta-analysis of other indicators showed that after treatment with TCM + conventional Western medicine, the serum total cholesterol concentration was lower, the duration of each angina attack was shorter, and the quality of life was better than that after treatment with conventional Western medicine alone, and the differences were statistically significant (all $\mathrm{P}<0.05$ ). However, no statistical significance was observed for other indicators.

\section{Adverse reaction rate}

As shown in Figure 6, only 2 studies reported the adverse reactions in the two treatment groups: Liu et al. (15) reported the occurrence of abnormal blood glucose and liver function damage, while Shi et al. (16) reported the occurrence of muscle spasm, arthralgia, and hepatitis in both groups. Meta-analysis revealed that the homogeneity of the two studies was good $\left(\mathrm{I}^{2}=0 \%, \mathrm{P}=0.41\right)$. The statistical effect size obtained by the fixed-effects model was [OR $=0.48,95 \%$ CI: $(0.21,1.08)]$ and the statistical effect value was $Z=1.78, P=0.08$; however, the difference was not statistically significant.

\section{Analysis of publication bias}

The funnel plot in Figure 7 shows uneven distribution between the two subgroups, suggesting the presence of 


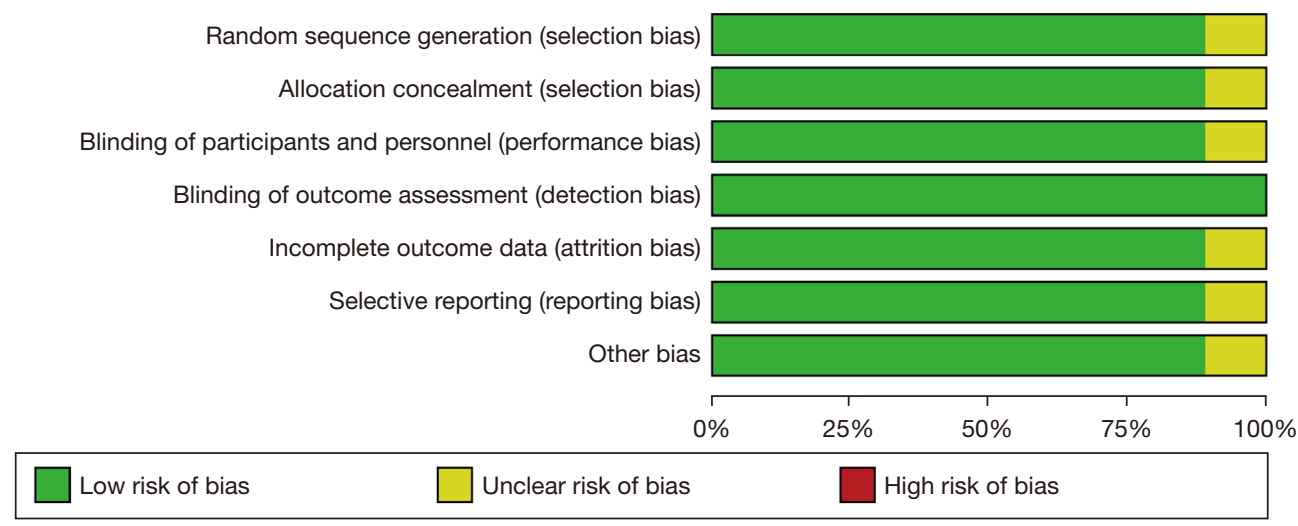

Figure 3 Risk-of-bias assessment of the 9 included articles.

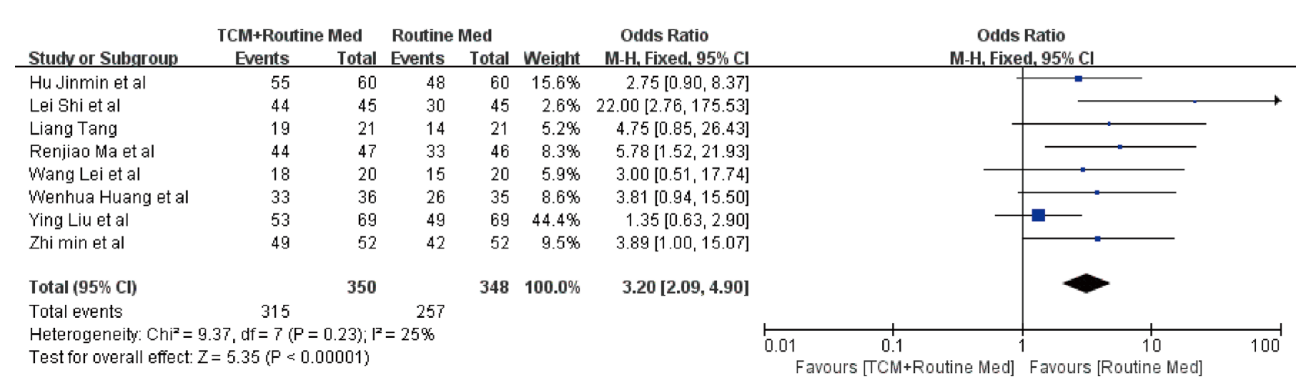

Figure 4 Comparison of the effective rate of angina pectoris using traditional Chinese medicine or conventional Western medicine.

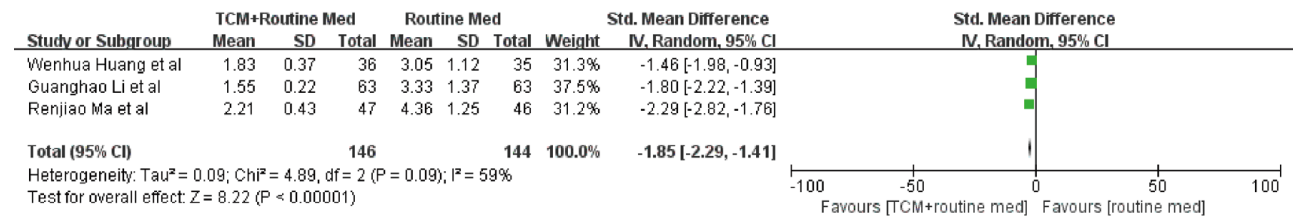

Figure 5 Comparison of angina pectoris attack frequency after treatment with traditional Chinese medicines for promoting blood circulation and removing blood stasis and conventional Western medicine.

Table 3 Meta-analysis of other outcome measures of the included studies (TCM + conventional Western medicine group and conventional Western medicine group)

\begin{tabular}{lcccl}
\hline Outcome measures & No. of articles & Effect size & $P$ value & Model \\
\hline Serum TC & 3 & $-0.53(-1.21,-0.31)$ & 0.007 & Random effects; $P<0.1 ; I^{2}=65 \%$ \\
TG & 3 & $-0.42(-0.84,-0.03)$ & 0.352 & Random effects; $P<0.1 ; I^{2}=78 \%$ \\
HDL-C & 3 & $-0.63(-0.99,-0.12)$ & 0.449 & Random effects; $P<0.1 ; I^{2}=56 \%$ \\
LDL-C & 3 & $0.19(0.08,1.55)$ & 0.241 & Random effects; $P<0.1 ; I^{2}=76 \%$ \\
Duration of each angina attack (min) & 2 & $-2.63(-3.65,-1.22)$ & 0.021 & Random effects; $P<0.1 ; I^{2}=55 \%$ \\
SAQ & 2 & $3.54(1.31,4.58)$ & 0.001 & Fixed effects; $P<0.1 ; I^{2}=15 \%$ \\
\hline
\end{tabular}

TC, total cholesterol; TG, triacylglycerol; HDL-C, high-density lipoprotein cholesterol; LDL-C, low-density lipoprotein cholesterol; SAQ, the Seattle Angina Questionnaire. 


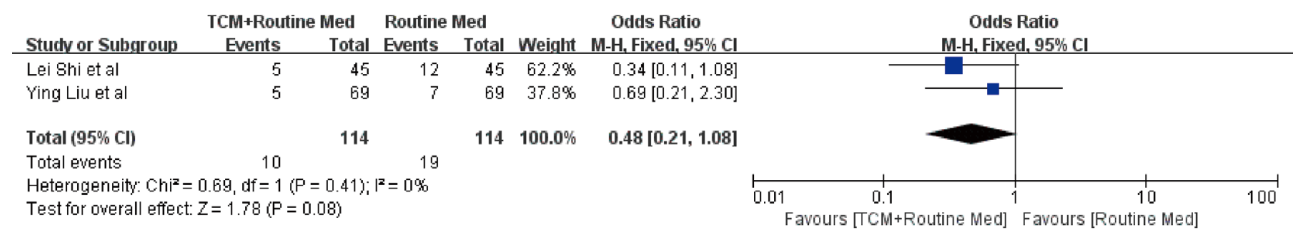

Figure 6 Comparison of adverse events between traditional Chinese medicines for promoting blood circulation and removing blood stasis and conventional Western medicine.

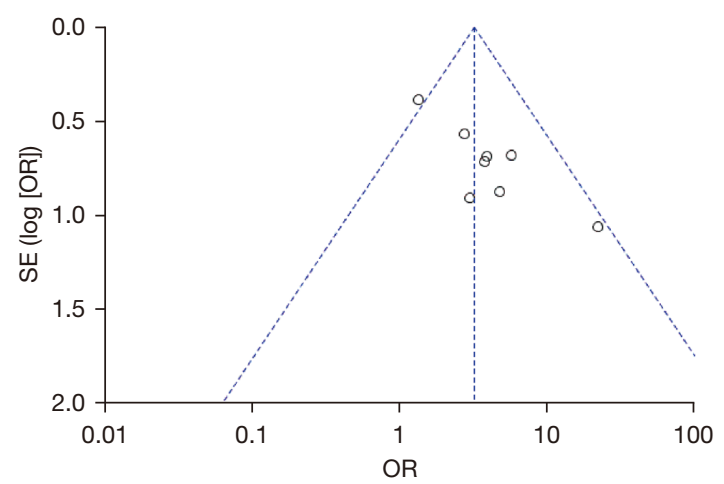

Figure 7 Funnel plot comparing traditional Chinese medicines for promoting blood circulation and removing blood stasis and conventional Western medicine treatment.

publication bias.

\section{Discussion}

The development of CHD may be related to a variety of factors such as sex, age, underlying diseases, drinking habits, and excessive obesity, all of which may lead to abnormal lipid metabolism. Such abnormality can damage the vascular endothelium and promote plaque formation and growth, resulting in stenosis and ischemic cardiomyopathy (17). In this systematic review and meta-analysis, 9 RCTs were included and a total number of 824 patients were involved. The results showed that the use of TCMs combining with conventional drugs for promoting blood circulation and removing blood stasis had the advantages of reducing the frequency of angina pectoris attacks, shortening the attack time, improving the therapeutic effect, reducing serum total cholesterol, and improving patient quality of life after treatment. Meanwhile, the two treatment approaches had equivalent efficacy in reducing the patients' blood lipids. Two of the nine studies reported on adverse reactions after treatment, and there was no significant difference in the incidence rate of adverse reactions between the two treatment methods which meant the TCM treatment won't add adverse reactions. The results of this study were consistent with those of the meta-analysis of Huang et al. (18), which included several (not reported) RCTs of TCMI (traditional Chinese medicine injection), but in this study, the experimental group interventions were administered through oral path, the results showed both administrating paths were effective.

The treatment of angina pectoris is mainly aimed at improving myocardial ischemia, reducing the frequency of attacks, and preventing the occurrence of myocardial infarction. Conventional medicines, such as nitrates, can improve the myocardial oxygen supply, protect myocardial cells, reduce the cardiac load, and delay the occurrence of cardiovascular disease. However, some patients cannot take conventional medicines for a long time due to insufficient drug tolerance (19).

Medicinal plant materials used in TCM, such as Panax notoginseng, Salvia miltiorrhiza, Hawthorn, Polygonum multiflorum, and Lonicera japonica, have been proved by modern pharmacology to regulate thrombosis, possess anti-oxidation and anti-aging properties, promote blood circulation, and remove blood stasis, ultimately helping to eliminate myocardial stasis and achieve the purpose of eliminating angina pectoris (20). Furthermore, TCMs have milder side effects than conventional medicines, making them more suitable for the long-term treatment of angina pectoris.

Also there are other TCM treatments clinically confirmed effective, a meta-analysis (21) of 12 RCTs showed that acupuncture therapy could serve as an adjunctive treatment of angina pectoris and contribute to the symptoms improving.

However, there were some limitations to this study. For instance, although the Jadad scores of the 9 included articles were all above 3 , but in some articles, the blinding or allocation concealment was unclear, which may have impacted on the results. Furthermore, although the 8 studies showed statistically homogeneity during synthesizing, the 
funnel plot showed uneven distribution, which suggested there might be the presence of publication bias.

Also, since few of the included studies reported indicators as blood lipid level, quality of life, and adverse events, only 2 or 3 literatures included during synthesis for these indicators, which might lead to unaccuracy of the results.

Individual studies reported indicators as serum inflammatory factor level, endothelial vascular function, or the hemorheological level, but no synthesis was performed due to few articles. Therefore, more RCTs are needed and included to investigate the effect of TCM on such indicators.

Furthermore, the formulas and compositions of the TCMs used in the 9 included RCTs differed, and there was no uniform standard for the conventional Western medicine treatment, which may have produced inconsistency when the two groups were compared. Still, more highquality studies are needed to continue in-depth discussion regarding the therapeutic effect of TCM on angina pectoris of CHD.

\section{Conclusions}

In this meta-analysis of the therapeutic effect of TCMs for promoting blood circulation and removing blood stasis on angina pectoris in patients with CHD, 9 studies involving 824 patients were included. The results showed that the application of TCM in the treatment of angina pectoris can improve the therapeutic effect, reduce the frequency of angina pectoris, shorten the attack time, reduce serum total cholesterol, and improve the quality of life after treatment; however, it has no significant effect on reducing blood lipids.

\section{Acknowledgments}

Funding: Natural Science Foundation of Zhejiang Province (grant No. LGF20H270013).

\section{Footnote}

Reporting Checklist: The authors have completed the PRISMA reporting checklist. Available at https://dx.doi. org/10.21037/apm-21-2233

Conflicts of Interest: All authors have completed the ICMJE uniform disclosure form (available at https://dx.doi. org/10.21037/apm-21-2233). The authors have no conflicts of interest to declare.

Ethical Statement: The authors are accountable for all aspects of the work in ensuring that questions related to the accuracy or integrity of any part of the work are appropriately investigated and resolved.

Open Access Statement: This is an Open Access article distributed in accordance with the Creative Commons Attribution-NonCommercial-NoDerivs 4.0 International License (CC BY-NC-ND 4.0), which permits the noncommercial replication and distribution of the article with the strict proviso that no changes or edits are made and the original work is properly cited (including links to both the formal publication through the relevant DOI and the license). See: https://creativecommons.org/licenses/by-nc-nd/4.0/.

\section{References}

1. Sanchis-Gomar F, Perez-Quilis C, Leischik R, et al. Epidemiology of coronary heart disease and acute coronary syndrome. Ann Transl Med 2016;4:256.

2. Deckers K, Schievink SHJ, Rodriquez MMF, et al. Coronary heart disease and risk for cognitive impairment or dementia: Systematic review and meta-analysis. PLoS One 2017;12:e0184244.

3. Xiong $\mathrm{X}$. Integrating traditional Chinese medicine into Western cardiovascular medicine: an evidence-based approach. Nat Rev Cardiol 2015;12:374.

4. Chao J, Dai Y, Verpoorte R, et al. Major achievements of evidence-based traditional Chinese medicine in treating major diseases. Biochem Pharmacol 2017;139:94-104.

5. Hao P, Jiang F, Cheng J, et al. Traditional Chinese Medicine for Cardiovascular Disease: Evidence and Potential Mechanisms. J Am Coll Cardiol 2017;69:2952-66.

6. Hu R. Clinical observation on the influence of Sini Decoction on cardiac contractility in coronary heart disease patients with angina pectoris. Tianjin Journal of Traditional Chinese Medicine 2004;1:28-30.

7. McCormick F, Cvetanovich GL, Kim JM, et al. An assessment of the quality of rotator cuff randomized controlled trials: utilizing the Jadad score and CONSORT criteria. J Shoulder Elbow Surg 2013;22:1180-5.

8. Ma R, Ni X, Shi B, et al. Yiqi Qiangxin Decoction Combined with atorvastatin in the treatment of 47 cases of unstable angina pectoris of coronary heart disease. Zhejiang Journal of Traditional Chinese Medicine 
2021;56:348.

9. Lei W, Fang YH. Clinical observation on 20 cases of stable angina pectoris of coronary heart disease with heart blood stasis treated by Compound Sanqi Humai decoction combined with Western Medicine. Journal of Gansu University of Traditional Chinese Medicine 2019;36:48-51.

10. Zhi MO, Kong Z. The Clinical Research into Coronary Heart Disease Angina Treated with Decoction for Nourishing Qi and Activating Blood Circulation and Removing Blood Stasis. Henan Traditional Chinese Medicine 2015;35:1787-9.

11. Hu J, Wang R, Yu X, et al. Clinical observation on 60 cases of unstable angina pectoris of coronary heart disease treated by Bushen Huayu Huatan method. New Chinese Traditional Medicine 2010;42:89-90.

12. Liang T. Study on the efficacy of integrated traditional Chinese and Western medicine in the treatment of stable angina pectoris of coronary heart disease. Electronic Journal of Integrated Cardiovascular Disease of Traditional Chinese and Western Medicine 2019;7:54.

13. Li G, Xu W, Cao M, et al. Clinical observation of Huangqi Baoxin Decoction in the treatment of coronary heart disease angina. Journal of traditional Shanghai University of Chinese Medicine 2019;33:20-3, 41.

14. Huang W, Xu Y, Jiang L, et al. Clinical observation on 36 cases of angina pectoris of coronary heart disease treated by modified Xuefu Zhuyu Decoction Combined with Western Medicine. Hunan Journal of Traditional Chinese Medicine 2018;34:7-9.

15. Liu Y, Ren M, Ji Q. Efficacy analysis of traditional integrated Chinese and Western medicine in the treatment of unstable angina pectoris of coronary heart disease. Journal of Changchun University of Traditional Chinese Medicine 2018;34:1202-4.

16. Shi L, Qin L, Wu Y, et al. Clinical observation of the combination of traditional Chinese and Western medicine in the treatment of unstable angina pectoris of coronary heart disease. Shenzhen Journal of Integrated Chinese and Western Medicine 2018;28:28-30.

17. Liu GP, Li GZ, Wang YL, et al. Modelling of inquiry diagnosis for coronary heart disease in Traditional Chinese Medicine by using multi-label learning. BMC Complement Altern Med 2010;10:37.

18. Huang L, Xu R, Huang X, et al. Traditional Chinese medicine injection for promoting blood circulation and removing blood stasis in treating angina pectoris of coronary heart disease: A protocol for systematic review and network meta-analysis. Medicine (Baltimore) 2021;100:e25608.

19. Wang X, Sun H, Zhang A, et al. Potential role of metabolomics approaches in the area of traditional Chinese medicine: as pillars of the bridge between Chinese and Western medicine. J Pharm Biomed Anal 2011;55:859-68.

20. Li S, Zhang B. Traditional Chinese medicine network pharmacology: theory, methodology and application. Chin J Nat Med 2013;11:110-20.

21. Liu Y, Meng HY, Khurwolah MR, et al. Acupuncture therapy for the treatment of stable angina pectoris: An updated meta-analysis of randomized controlled trials. Complement Ther Clin Pract 2019;34:247-53.

(English Language Editor: J. Reynolds)
Cite this article as: Chen W, Wang B, Ge Y, Xu H, Jiang C, Yu P, Zhang X, Yang J, Hu H, Song H. A systematic review and meta-analysis of clinical research on treating angina pectoris of coronary heart disease with traditional Chinese medicine to promote blood circulation and remove blood stasis. Ann Palliat Med 2021;10(10):10506-10514. doi: 10.21037/apm-21-2233 